\title{
Microstructural analysis of negative anticipatory contrast: A reconsideration of the devaluation account
}

\author{
Rebecca L. Wright • Gary Gilmour • \\ Dominic M. Dwyer
}

Published online: 18 May 2013

(C) Psychonomic Society, Inc. 2013

\begin{abstract}
An animal's appetitive behavior is not a fixed response to current stimulation but can be affected by the anticipation of future events. For example, rats regularly given access to a moderately valued solution followed by a higher value solution (e.g., $4 \%$ sucrose $\rightarrow 32 \%$ sucrose) consume less of the initial solution than in control conditions where the initial solution is not followed by a higher value solution (e.g., $4 \%$ sucrose $\rightarrow 4 \%$ sucrose). Previous analyses have suggested that this negative anticipatory contrast effect does not depend on the "expectation" of a valuable stimulus producing a functional devaluation of a currently available stimulus of lesser value. In a withinsubjects anticipatory contrast procedure, this study revealed that both consumption and the mean size of licking clusters were smaller for a $4 \%$ sucrose solution on days when it preceded $32 \%$ sucrose than on days when $4 \%$ preceded $4 \%$. Since lick cluster size typically bears a positive monotonic relationship with the concentration of palatable solutions, this reduction is indicative of a decrease in the palatability/hedonic value of the solution subject to contrast. As such, we provide direct evidence that negative anticipatory contrast does produce a functional devaluation of the solution, thus challenging prevailing theoretical assumptions.
\end{abstract}

Keywords Contrast · Licking · Microstructural analysis · Cluster size $\cdot$ Rats

R. L. Wright • D. M. Dwyer $(\square)$

School of Psychology, Cardiff University,

Tower Building, Park Place,

Cardiff CF10 3AT, UK

e-mail: DwyerDM@cardiff.ac.uk

G. Gilmour

Eli Lilly \& Co. Ltd., Lilly Research Centre,

Erl Wood Manor, Sunninghill Road, Windlesham,

Surrey GU20 6PH, UK
An animal's behavior toward food is not fixed but can be adjusted to take into account future rewarding events. For example, a rat given brief daily sequential access to two solutions will learn to expect the second upcoming solution and will adjust its consumption of the currently available solution accordingly. If the second solution is preferred over the first, intake of the first solution will be suppressed (e.g., Flaherty, Coppotelli, Grigson, Mitchell, \& Flaherty, 1995). This modification of current behavior as a consequence of future exposure to alternative stimuli is known as anticipatory contrast. The suppression of the first solutions intake has been ascribed to a contrast effect based on the comparison between the levels of reward available at the time and the level of reward expected in the near future. This suppression appears to be genuinely anticipatory because, in within-subjects designs, intake from the first bottle available in a day is low when the upcoming solution is valuable, while the value of the solution consumed the previous day has little effect (Flaherty et al., 1995; Flaherty \& Rowan, 1985). More generally, the existence of within-subjects anticipatory contrast demonstrates that the effect cannot simply be due to a comparison between the currently available solution and the animal's previous overall experience. Furthermore, increasing the interval between solutions within a day reduces contrast, which would not be the case if the reduction in consumption was based simply on comparison with previous experience in that context (e.g., Flaherty \& Checke, 1982; Lucas, Gawley, \& Timberlake, 1988).

While the behavioral phenomenon of anticipatory contrast is well established, few mechanisms have been put forward to explain how current behavior can be suppressed by the expectation of a more rewarding event. Flaherty and Rowan (1985) proposed that exposure to the first solution, together with the context of its presentation, allows a comparison between the different solution values by invoking an internal representation of the impending preferred solution. Flaherty (1996) considered three general mechanisms by 
which this might lead to a reduction in consumption of the first solution: a relative devaluation of the first solution; spatial competition from goal tracking (i.e., the animal repeatedly approaches the location of the not-yet accessible second solution); or response inhibition, where the animal learns to inhibit intake of the first solution because the second solution is more rewarding (see also Flaherty et al., 1995; Onishi \& Xavier, 2011). Of these, the devaluation account is perhaps the most plausible. This account dictates that as an animal learns to expect a valuable stimulus in the near future, there is a functional devaluation of the currently available reinforcer of lesser value - hence, a lower consummatory response.

While intuitively plausible, the devaluation account appears to be inconsistent with some previous results. Indeed, Weatherly, Nurnberger, and Sturdevant (2006) found that a $1 \%$ sucrose solution subject to anticipatory contrast did not suffer a reduction in its ability to act as a reinforcer for operant behavior, as compared with a noncontrasted $1 \%$ solution of sucrose. Furthermore, when different spout cues were paired with contrasted and noncontrasted solutions, Flaherty et al. (1995) found that cues paired with the contrasted substance were not avoided in preference tests, as compared with cues paired with the control (noncontrasted) substance. The fact that solutions subject to anticipatory contrast do not suffer a reduction in their ability to act as reinforcers in either instrumental (Weatherly et al., 2006) or Pavlovian (Flaherty et al., 1995) situations would seem to suggest that their rewarding value has not been diminished by being reliably presented in advance of a preferred solution.

That said, neither Weatherly et al. (2006) nor Flaherty et al. (1995) actually assessed the value of the solution subject to contrast, so they do not directly demonstrate that the value of the contrasted solution is maintained. More important, the nature of the anticipatory contrast procedure means that the contrasted substance is a perfectly reliable cue for a highly rewarding event. It has long been known that otherwise neutral cues paired with rewarding events can themselves support instrumental or Pavlovian conditioning as secondary reinforcers (see Mackintosh, 1974). Thus, a solution subject to contrast might have supported subsequent responses as a secondary, rather than a primary, reinforcer even if anticipatory contrast had reduced the intrinsic value of the initial solution itself. Direct measurement of the hedonic response to the solution subject to contrast would address these issues.

One method for directly assessing the hedonic value of a solution is to analyze the microstructure of licking displayed by a rat during voluntary consumption. When drinking, rats do not lick continuously but perform repeated runs of licks (herein referred to as clusters) separated by pauses of varying length. When consuming sucrose (and other palatable solutions), the mean number of licks per cluster (lick cluster size) bears a positive monotonic relationship with the solution concentration (e.g., Davis \& Smith, 1992; Spector, Klumpp, \& Kaplan, 1998). Conversely, when rats consume an unpalatable solution (such as quinine), lick cluster size bears a negative monotonic relationship with the concentration of the solution (Hsiao \& Fan, 1993; Spector \& St John, 1998). This has led to the idea that lick cluster size measures can be used as a reliable index of stimulus palatability and, hence, the affective component of reward value (see Dwyer, 2012, for a recent review). Critically, lick cluster size is at least partially independent of consumption, which typically displays an inverted U-shaped function between concentration and total consumption, with intermediate concentrations of palatable solutions eliciting the highest levels of intake (e.g., Richter \& Campbell, 1940). As reviewed by Dwyer (2012), the dissociation between the two measures has been confirmed through flavor preference and aversion conditioning studies, which have demonstrated that some manipulations can influence lick cluster sizes, while leaving total consumption unchanged, and vice versa. Furthermore, it has been demonstrated that some taste aversions result in changes in lick cluster size that are analogous to actually changing a pleasant-tasting solution to an aversive one, while flavor preference conditioning result in changes that are analogous to changing the solution from a neutral to a palatable taste. With lick cluster sizes typically reflecting the nature of the solution, changes that occur when the solution itself is physically unaltered suggest that the change must lie with the animal. That is, it is the animals' perception or evaluation of the solution that has changed (see Dwyer, 2012).

There has been one study to our knowledge that combined lick microstructure measures with an anticipatory contrast paradigm. Arthurs, Lin, Amodeo, and Reilly (2012) found a difference in lick cluster size for a saccharin solution as a factor of whether it was followed by higher valued sucrose or more of the same solution. Such suppressed lick cluster sizes appear, as far as we are concerned, to be wholly consistent with a reduction in the first solution's rewarding value, relative to appropriate controls. However, the analysis of the results offered by Arthurs et al. led them to conclude that this difference was not, in fact, a product of devaluation (we will address these differences of interpretation more fully in the Discussion section). Be that as it may, it should be noted that the Arthurs et al. study used a between-subjects design, which means that animals in the contrast and control conditions differed in their exposure to concentrated sucrose. Repeated exposure to concentrated sucrose in the contrast group could have resulted in a shift in their general adaptation levels to sweet and, thus, lowered their sensitivity to the relatively weak sweet taste of dilute saccharin (Albertella, Harris, \& Boakes, 
2008; Boakes, Albertella, \& Harris, 2007). Although general differences in experience with different concentrations of sucrose cannot explain all previously observed anticipatory contrast effects (see the comments above regarding withinsubjects and intersolution time effects), it remains the case that the suppressed lick cluster sizes observed by Arthurs et al. may reflect differences in overall experience, rather than being the product of anticipatory contrast.

The present study used a within-subjects design to address the reliability and source of cluster size changes in anticipatory contrast. Importantly, in this design, all animals received exposure to all test solutions, eliminating any differences in the level or type of solution exposure. Different contextual cues (chosen on the basis of the work of Flaherty et al., 1995) acted to signal which of the two solution pairings (either a low-reward solution followed by more of the same solution or a low-reward solution followed by a high-reward solution) was in operation each day. We reasoned that if the reduction in consumption (i.e., anticipatory negative contrast) of the initial solution when it precedes a preferred solution occurs because the first solution is devalued, it will be mirrored by a similar reduction in lick cluster size.

\section{Method}

\section{Subjects and apparatus}

Male Lister-hooded rats $(n=8$, Harlan, U.K.), weighing 300-340 $\mathrm{g}$ on ad lib food, were used in the experiment. They were paired-housed, under a 12:12-h light:dark cycle. Experimental sessions were performed during the light phase, beginning at approximately 11 a.m., and were conducted 6-7 days per week. Prior to the start of the experiment, all animals were placed on a food-restricted diet, which maintained them between $85 \%$ and $95 \%$ of their free-feeding weights. Their food ration was given in their home cage $30 \mathrm{~min}$ after the end of each daily session. The experiment was conducted in accordance with the United Kingdom Animals Scientific Procedures Act, 1986.

Testing was conducted in six automated drinking chambers (Med Associates Inc., St Albans, VT), measuring $30 \times$ $24 \times 21 \mathrm{~cm}$ and consisting of two clear Perspex and two aluminum walls. The chamber floor consisted of 19 steel rods, $4.8 \mathrm{~mm}$ in diameter and $16 \mathrm{~mm}$ apart. Approximately $5 \mathrm{~cm}$ above the grid floor, two holes, each of $1-\mathrm{cm}$ diameter, were positioned on each side of one aluminum wall to allow the rat access to the solutions. Solutions were delivered through the right and left access holes by $50-\mathrm{ml}$ cylinders with ball-bearing metal drinking spouts. These were mounted to the cage via motorized holders that held the spout flush with the outside of the chamber and retracted it as required. Contact-sensitive lickometers registered the timing of each lick made by the animal to the nearest $0.01 \mathrm{~s}$, and a computer running MED-PC software controlled the equipment and recorded the data. The solutions used were $4 \%$ and $32 \%$ (wt/wt) sucrose formulated using commercial-grade cane sugar and deionized water.

\section{Procedure}

On the first day of the experiment, the animals (which had been water deprived for $22 \mathrm{~h}$ ) were habituated by leaving them in the drinking chambers with 10-min access to water from both bottles. After this pretraining, the animals were returned to an ad lib water supply for the remainder of the experiment. On each subsequent training day, the solution pairings were manipulated within subjects. Rats were presented with either a $4 \%$ sucrose solution followed by more $4 \%$ sucrose (the $4-4$ condition) or a $4 \%$ sucrose solution followed by a $32 \%$ sucrose solution (the 4-32 condition). These daily solution pairings were presented in double alternation (e.g., ABBAABBA), and different contextual cues were used to signal which of the two solution pairings was in operation each day. For half the animals, context 1 (consisting of bright light, white noise provided by a detuned radio, and a normal grid floor) was paired with the 4-4 condition, and context 2 (consisting of dim light provided by a table lamp, no background noise, and a wire mesh floor insert) was paired with the 4-32 condition. The remaining subjects had the opposite pairings. The first solution in the pair was made available for $3 \mathrm{~min}$ on the lefthand side of the chamber. Following a 4-sec intersolution interval, the second solution was then made available for 6 min on the right-hand side of the chamber.

\section{Data analysis}

Consumption was assessed by weighing the bottle before and after each experimental run. Lick cluster size (defined as the mean number of licks per cluster) was extracted from the MED-PC data. As in our previous experiments using these general methods and equipment (e.g., Dwyer, Lydall, \& Hayward, 2011; Lydall, Gilmour, \& Dwyer, 2010), a cluster was defined as a series of licks, with each lick separated by no more than a 0.5 -s interval. The same criterion had been adopted by Davis and his colleagues (Davis, 1989; Davis \& Perez, 1993; Davis \& Smith, 1992). Although other criteria have been used (e.g., $1 \mathrm{~s}$ by Spector et al., 1998), there is little practical difference, since most pauses greater than $0.5 \mathrm{~s}$ are also greater than $1 \mathrm{~s}$ (Davis \& Smith, 1992; Spector et al., 1998). Drinking data were collated into twosession blocks. An alpha level of .05 was adopted as the level of significance throughout. 


\section{Results}

Figure 1 depicts the consumption (panel A) and lick cluster size measures (panel B), across the eight 2-session blocks, of the initial $4 \%$ solution as a factor of whether it was followed by $4 \%$ sucrose (the $4-4$ condition) or $32 \%$ sucrose (the 4-32 condition). Inspection of Fig. 1a suggests that intake of the initial $4 \%$ solution increased across blocks to a greater extent for the 4-4 condition than for the 4-32 condition, representing an anticipatory contrast effect. A repeated measures ANOVA with factors of block (1-8) and contrast condition (4-4 vs. 4-32) revealed a nonsignificant main effect of contrast condition, $F(1,7)=2.79, p=.139$, $M S E=1.13$, a significant main effect of block, $F(7,49)=$ 17.88, $p<.001, M S E=6.27$, and a significant contrast condition $\times$ block interaction, $F(7,49)=2.31, p=.041$, $M S E=0.28$. A post hoc analysis of the interaction suggests no difference between the 4-4 and 4-32 conditions for blocks $1,2,3$, and 5 [largest $F(1,7)=1.18, p=.314$,
$M S E=0.05$, for block 2], while there were significant differences for blocks $4,6,7$, and 8 [smallest $F(1,7)=$ $5.76, p=.048, M S E=0.01$, for block 7].

An inspection of Fig. 1b indicates that the anticipatory contrast effect on consumption was associated with lower lick cluster sizes in the contrasted (4-32) than in the noncontrasted (4-4) condition during intake of the initial solution. An ANOVA revealed a significant main effect of contrast condition, $F(1,7)=24.57, p=.002, M S E=$ 1,203.07, a significant main effect of block, $F(7,49)=$ 7.11, $p<.001, M S E=764.93$, and a significant contrast condition $\times$ block interaction, $F(7,49)=3.70, p=.003$, $M S E=189.50$. A follow-up analysis revealed no significant differences between contrast conditions during blocks 1, 2, 3,4 , and 7 [largest $F(1,7)=5.45, p=.052, M S E=11.10$, for block 4] and showed that there were significant differences in blocks 5, 6, and 8 [smallest $F(1,7)=7.22, p=.031$, $M S E=15.27$, for block 6]. This result indicates that pairing $4 \%$ sucrose with $32 \%$ sucrose suppresses the increase in
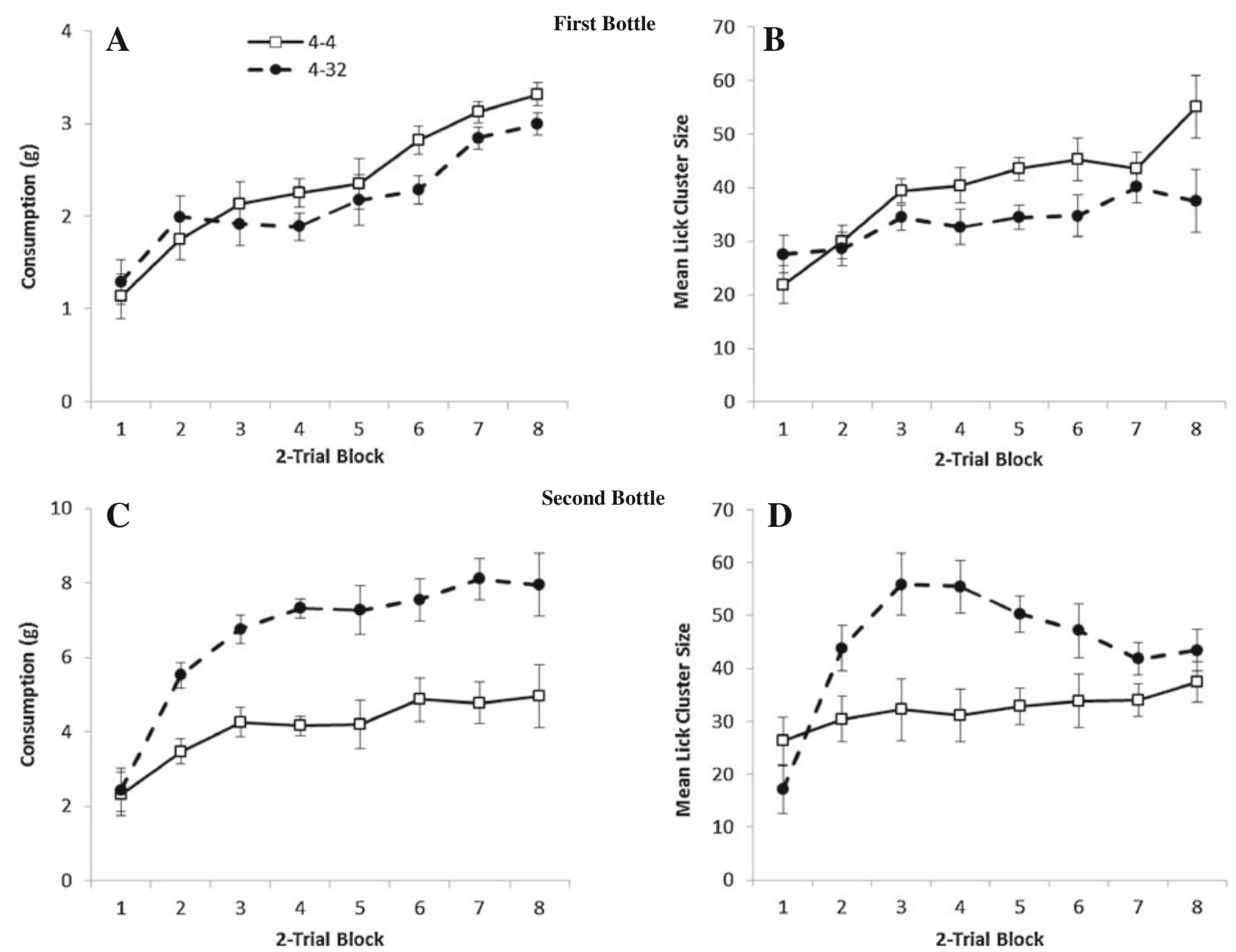

Fig. 1 a Mean ( $\pm S E$ of the difference) consumption data from the first bottle available (containing $4 \%$ sucrose) each day during negative anticipatory contrast for the 4-4 (control) and 4-32 (contrast) conditions. b Mean lick cluster size ( $\pm S E$ of the difference) for the first bottle available each day as a factor of contrast condition. The first bottle was available for $3 \mathrm{~min}$. c Mean ( $\pm S E$ of the difference) consumption data

from the second bottle available each day during negative anticipatory contrast for the 4-4 (control) and 4-32 (contrast) conditions. d Mean lick cluster size ( $\pm S E$ of the difference) for the second bottle available each day as a factor of contrast condition (4-4 vs. 4-32). The second bottle was available for 6 min (beginning $4 \mathrm{~s}$ after the first bottle had been retracted). The data are averaged over two trial blocks 
lick cluster size that would have otherwise occurred if it had been followed by more of the same solution (the 4-4 condition).

Figure $1 \mathrm{c}$ and $\mathrm{d}$ show the consumption and lick cluster size measures, respectively, for the second sucrose solution in conditions 4-4 and 4-32 over the eight 2-day blocks of the experiment. As can be seen in panel $\mathrm{C}$, the consumption of the second solution ( $4 \%$ sucrose) remained consistently low across blocks for the 4-4 condition. In contrast, the consumption of the second solution ( $32 \%$ sucrose) in the 432 condition increased over the blocks. An ANOVA revealed significant main effects of contrast condition, $F(1$, $7)=39.17, p<.001, M S E=198.25$, and of block, $F(7,49)=$ 38.37, $p<.001, M S E=29.82$, and an interaction between them, $F(7,49)=6.46, p<.001, M S E=4.33$. Post hoc tests showed that there was no significant difference between conditions at block $1, F<1$, while there were significant differences at blocks $2-8$ [smallest $F(1,7)=12.74, p=.009$, $M S E=0.70$, for block 8]. Inspection of panel D reveals a similar pattern of results for lick cluster size in that the lick clusters were consistently higher, at least numerically so, for the 4-32 than for the 4-4 condition across blocks. An ANOVA revealed significant main effects of contrast condition, $F(1,7)=31.39, p<.001, M S E=4664.57$, and block, $F(7,49)=14.96, p<.001, M S E=813.67$, plus a significant contrast condition $\times$ block interaction, $F(7,49)=6.74, p<$ $.001, M S E=470.47$. Post hoc analysis revealed no significant difference between conditions at block $1, F(1,7)=$ 4.177, $p=.080, M S E=20.14$, significant differences in blocks 2-7 [smallest $F(1,7)=6.55, p=.038, M S E=9.23$, for block 7], but no difference in block $8, F(1,7)=2.345, p$ $=.170, M S E=15.09$. This pattern of effects appears to be largely driven by the gradual reduction in lick cluster sizes during consumption of $32 \%$ sucrose from block 4 to 8 . The reason for this downward trend is not clear; however, we have also observed similar reductions across exposure sessions when animals were repeatedly presented with sucrose in the absence of an anticipatory contrast procedure. It is possible that it might reflect within-session adaptation to the concentrated sucrose (Dwyer, 2012) that is exacerbated as consumption levels increase. ${ }^{1}$

\footnotetext{
${ }^{1}$ It is also possible that the reduced lick cluster size seen for the $32 \%$ solution across training is due to sucrose-induced insulin resistance. High-sucrose diets have previously been shown to impair insulin action in rats (e.g., Storlien, Kraegen, Jenkins, \& Chisholm, 1988). Furthermore, Ribeiro, Lautt, Legare, and Macedo (2005) gave Sprague-Dawley rats free access to a $35 \%$ sucrose solution (along with food and water ad lib) and found that insulin resistance was expressed as early as 2 weeks in this strain. Since exposure to concentrated sucrose is restricted to $6 \mathrm{~min}$ a day in our paradigm, this possibility may be unlikely but cannot be ruled out on the basis of the present data alone.
}

\section{Discussion}

In one context, rats received access to $4 \%$ sucrose from one bottle followed by access to $4 \%$ sucrose from a second bottle, while in a different context, they received access to $4 \%$ sucrose from one bottle followed by access to $32 \%$ sucrose from a second bottle. The rats' consumption of $4 \%$ sucrose was lower on days when $4 \%$ sucrose preceded access to $32 \%$ sucrose than when it preceded access to more $4 \%$ sucrose. This reflects a within-subjects anticipatory contrast effect on consumption. Moreover, an analysis of licking microstructure revealed that this contrast effect was also reflected in the size of licking clusters. That is, the same $4 \%$ sucrose elicited lower lick cluster sizes on days when it was followed by $32 \%$ sucrose than on days when it was followed by $4 \%$ sucrose. Since lick cluster size is directly related to the perceived value or concentration of sucrose and the first solution was physically unchanged, this effect is consistent with anticipatory contrast producing a devaluation of $4 \%$ sucrose relative to an appropriate control. That is to say, the differences in the mean number of licks per cluster between the 4-4 and 4-32 conditions result from a change in the perceived value of the initial solution by the anticipation of future rewards. Moreover, because a within-subjects procedure was used, the effects observed here cannot be attributed to a general reduction in the sensitivity to sweet tastes as a result of adapting to high sucrose concentrations. Contrary to the majority of previous analyses, this leads us to suggest that negative anticipatory contrast does indeed result in a devaluation of the initial solution.

While the use of a within-subjects design means that the suppressed lick cluster sizes observed cannot be attributed to a general reduction in the rat's sensitivity to sweetness as a result of shifts in their overall adaptation level (Boakes et al., 2007), there is evidence for context-specific adaptationlevel effects (Albertella et al., 2008). In this light, it is thus possible that the reduced lick cluster sizes are due to a comparison between the concentration of sucrose previously experienced in a particular context and the currently available solution, rather than being the product of an anticipatory comparison process. That said, it should be remembered that the interval between two solutions within a day influences consumption effects in anticipatory contrast (e.g., Flaherty \& Checke, 1982; Lucas et al., 1988). This timing effect would not be expected if anticipatory contrast were actually due to a comparison between the currently available solution and the stored value of previous solutions experienced in the same context. Since we have not manipulated intersolution intervals here, we cannot directly rule out the possibility that context-dependent adaptation effects contributed to our lick-microstructure results, and so our lick-microstructure and consumption results might reflect 
different causal mechanisms. However, we would suggest that it is more parsimonious to assume that contrast effects on consumption and on lick cluster size share a common cause. This is especially so given that the effects of contrast on consumption and lick cluster size emerged at roughly the same point in the experiment.

The idea that the lower lick cluster sizes for $4 \%$ sucrose in the 4-32 than in the 4-4 condition reflects devaluation in the former condition might seem to be a relatively direct corollary of the generally observed relationship between lick cluster size and solution concentration or value. However, Arthurs et al. (2012) previously reported similar results from a between-subjects design, while concluding that devaluation was not involved. This conclusion was based on the fact that, in animals for which saccharin preceded sucrose, the cluster size for saccharin remained relatively consistent across training, while in animals for which saccharin preceded further saccharin access, the cluster size for saccharin increased across sessions. That is, there was no evidence from the lick cluster size measure that the value of saccharin reduced from its initial level as a result of anticipatory contrast (essentially the same pattern of results was observed here with $4 \%$ sucrose). However, it should be remembered that rodents typically show a neophobic response to novel tastes that dissipates with experience. Indeed, Lin, Amodeo, Arthurs, and Reilly (2012) reported that lick cluster sizes increase over exposure for a variety of solutions and similar results were seen by Dwyer (2009). Lin et al. neatly summarized that the clear implication of these results is that "the pleasure of drinking increases as the novel, potentially dangerous tastant becomes accepted as safe" (p. 515). In this light, the failure to see an increase in the lick cluster size for saccharin (by Arthurs et al., 2012) or $4 \%$ sucrose (here) as a result of anticipatory contrast does represent a devaluation, relative to the state that would have occurred had the solution simply been exposed on its own. To be sure, pairing saccharin or sucrose with illness can produce devaluations relative to the initial state (e.g., Arthurs et al., 2012; Dwyer, 2009), but the mere fact that other treatments produce larger effects does not mean that contrast is not producing a devaluation at all.

A devaluation account of anticipatory contrast seems intuitively plausible: The decrease in responding for a lowvalued solution when a high-valued solution will be available in the near future occurs because the initial solution has become one of functionally lower hedonic value. However, this devaluation interpretation has generally been rejected, largely because solutions that have been subject to anticipatory contrast appear to operate as positive rewards in both instrumental (e.g., Weatherly et al., 2006) and Pavlovian (e.g., Flaherty et al., 1995) situations. But, as was noted in the introduction, these are not direct tests of the functional value of the solution subject to contrast, and more critically, the reinforcing value of the contrasted solutions could be attributed to a process of secondary reinforcement. Since the present study directly addressed the value of the contrasted solution via the analysis of licking microstructure and did see a functional devaluation, it would appear that previous theorists might have been premature in rejecting the devaluation account.

To summarize, the present study is the first to combine microstructural lick analysis with a within-subjects negative anticipatory contrast procedure and, thus, avoids the problems either of using indirect assessments of reward value or of confounds relating to adaptation level to sweet tastes between groups. The results obtained confirm, contrary to prevailing assumptions, that anticipatory contrast does produce a functional devaluation of the solution subject to contrast. What remains to be ascertained is whether this devaluation is the cause of the reduction in the amount of consumption also seen in contrast, or whether the changes in solution value and amount consumed are independent effects of experiencing contrast.

Author Note This research was supported by a BBSRC CASE studentship awarded to R.W. and cofunded by Eli Lilly \& Co.

\section{References}

Albertella, L., Harris, J. A., \& Boakes, R. A. (2008). Acquired flavor preferences: Contextual control of adaptation-level effects. Quarterly Journal of Experimental Psychology, 61, 227-231.

Arthurs, J., Lin, J.-Y., Amodeo, L. R., \& Reilly, S. (2012). Reduced palatability in drug-induced taste aversion: II. Aversive and rewarding unconditioned stimuli. Behavioral Neuroscience, 126, 433-444.

Boakes, R. A., Albertella, L., \& Harris, J. A. (2007). Expression of flavor preference depends on type of test and on recent drinking history. Journal of Experimental Psychology. Animal Behavior Processes, 33, 327-338.

Davis, J. D. (1989). The microstructure of ingestive behavior. Annals of the New York Academy of Sciences, 575, 106-121.

Davis, J. D., \& Perez, M. C. (1993). Food deprivation-induced and palatability-induced microstructural changes in ingestive behavior. American Journal of Physiology, 264, R97-R103.

Davis, J. D., \& Smith, G. P. (1992). Analysis of the microstructure of the rhythmic tongue movements of rats ingesting maltose and sucrose solutions. Behavioral Neuroscience, 106, 217-228.

Dwyer, D. M. (2009). Microstructural analysis of ingestive behaviour reveals no contribution of palatability to the incomplete extinction of a conditioned taste aversion. Quarterly Journal of Experimental Psychology, 62, 9-17.

Dwyer, D. M. (2012). Licking and liking: The assessment of hedonic responses in rodents. Quarterly Journal of Experimental Psychology, 65, 371-394.

Dwyer, D. M., Lydall, E. S., \& Hayward, A. J. (2011). Simultaneous contrast: Evidence from licking microstructure and cross-solution comparisons. Journal of Experimental Psychology. Animal Behavior Processes, 37, 200-210.

Flaherty, C. F. (1996). Incentive relativity. Cambridge: Cambridge University Press. 
Flaherty, C. F., \& Checke, S. (1982). Anticipation of incentive gain. Learning \& Behavior, 10, 177-182.

Flaherty, C. F., Coppotelli, C., Grigson, P. S., Mitchell, C., \& Flaherty, J. E. (1995). Investigation of the devaluation interpretation of anticipatory negative contrast. Journal of Experimental Psychology. Animal Behavior Processes, 21, 229-247.

Flaherty, C. F., \& Rowan, G. (1985). Anticipatory contrast: Withinsubjects analysis. Learning \& Behavior, 13, 2-5.

Hsiao, S., \& Fan, R. J. (1993). Additivity of taste-specific effects of sucrose and quinine: Microstructural analysis of ingestive behavior in rats. Behavioral Neuroscience, 107, 317-326.

Lin, J. Y., Amodeo, L. R., Arthurs, J., \& Reilly, S. (2012). Taste neophobia and palatability: The pleasure of drinking. Physiology and Behavior, 106, 515-519.

Lucas, G. A., Gawley, D. J., \& Timberlake, W. (1988). Anticipatory Contrast as a measure of time horizons in the rat: Some methodological determinants. Animal Learning and Behavior, 16, 377382.

Lydall, E. S., Gilmour, G., \& Dwyer, D. M. (2010). Analysis of licking microstructure provides no evidence for a reduction in reward value following acute or sub-chronic phencyclidine administration. Psychopharmacology, 209, 153-162.

Mackintosh, N. J. (1974) The psychology of animal learning. London: Academic.
Onishi, B. K. A., \& Xavier, G. F. (2011). Negative anticipatory contrast: Does it involve anticipation of an impending reward? Behavioural Processes, 86, 263-271.

Ribeiro, R. T., Lautt, W. W., Legare, D. J., \& Macedo, M. P. (2005). Insulin resistance induced by sucrose feeding in rats is due to an impairment of the hepatic parasympathetic nerves. Diabetologia, 48, 976-983.

Richter, C. P., \& Campbell, K. H. (1940). Taste thresholds and taste preferences of rats for five common sugars. Journal of Nutrition, $20,31-46$

Spector, A. C., Klumpp, P. A., \& Kaplan, J. M. (1998). Analytical issues in the evaluation of food deprivation and sucrose concentration effects on the microstructure of licking behavior in the rat. Behavioral Neuroscience, 112, 678-694.

Spector, A. C., \& St John, S. J. (1998). Role of taste in the microstructure of quinine ingestion by rats. American Journal of Physiology, 274, R1687-R1703.

Storlien, L. H., Kraegen, E. W., Jenkins, A. B., \& Chisholm, D. J. (1988). Effects of sucrose vs starch diets on in vivo insulin action, thermogenesis, and obesity in rats. The American Journal of Clinical Nutrition, 47, 420-427.

Weatherly, J. N., Nurnberger, J. T., \& Sturdevant, M. (2006). Investigating the devaluation explanation for negative anticipatory contrast. Journal of Experimental Psychology. Animal Behavior Processes, 32, 102-107. 